UDK 821.131.1-32.09Groto L.:133.52:929Boccaccio G.

\title{
ELEMENTI DI ASTROLOGIA NEL DECAMERON CENSURATO DI LUIGI GROTO
}

\author{
Kristina Lazar
}

\begin{abstract}
The censored version of the Decameron by Luigi Groto, which was published in 1588, was already the third censorship of Boccaccio's masterpiece during the Counter-Reformation. As opposed to the other two censured versions, this one did not place emphasis on philological questions, but it tried above all to narratively fill the gaps created by expurgation; in some cases following the original plot and in the others changing it to such a degree that the novels remained barely recognizable. This article exposes some characteristics of Groto's mannerist style; especially the elements of astrology, which emerge in the expurgated parts of the text, and the way these elements function when inserted into Boccaccio's text.
\end{abstract}

Key words: Controriforma, censura libraria, Decameron, Giovanni Boccaccio, Luigi Grota, Astrologia

\section{SFONDO STORICO DELLA VERSIONE GROTIANA}

La versione censurata del Decameron a cura di Luigi Groto, che uscì nel 1588, era già la terza rassettatura del capolavoro boccacciano nell'arco di quindici anni (la versione dei Deputati era uscita nel 1573 e quella di Lionardo Salviati nel 1582). Anche se pubblicata come ultima, era stata scritta già parecchi anni prima, nel 1579 , solo sei mesi dopo che il Groto ne aveva ricevuto l'incarico, e subito dopo mandata in Vaticano, dove in seguito fu smarrita (non si è appurato se casualmente o no). Solo nel $1584 \mathrm{fu}$ ritrovato il libro a stampa sul quale il Groto aveva segnato i brani da espurgare, mentre del manoscritto che conteneva la nuova versione delle parti eliminate non si sa niente (Chiecchi 1984: 88-90). La questione ancor oggi non definitivamente risolta è quale sia dunque il testo pubblicato a Venezia nel 1588, se quello del manoscritto consegnato e poi smarrito $^{1}$ - oppure, come più probabile, uno nuovo, scritto nell'arco di un anno e mezzo prima della sua morte, avvenuta nel dicembre 1585. La rassettatura curata dal Groto fu infatti pubblicata postuma per iniziativa di Giovanni Sega, marito di Claudia Groto, cugina

\footnotetext{
${ }^{1}$ Questo però ci sembra poco probabile, visto che l'autore di solito ringraziava oltremodo per ogni favore, nelle sue lettere (Groto 2007), e in questo caso non dice niente a proposito.
} 
ed erede di Luigi (Chiecchi 1984: 85). Questa è però solo una tra le questioni irrisolte che riguardano la censura grotiana. Assai significativa sembra la questione dello smarrimento della sua opera a Roma, che aperse la strada ad un nuovo incarico di rassettatura, stavolta assegnato ad un personaggio ben più influente del Groto, il futuro cruscante Salviati. Non sembra improbabile che Salviati avesse concorso lui stesso a ricevere questo prestigioso lavoro e, suffragato dal duca di Sora, figlio del pontefice Gregorio XII (Chiecchi 1984: 89), con l'aiuto di alte conoscenze abbia fatto sì che il manoscritto grotiano andasse perso. ${ }^{2}$ A causa della mancanza di documenti, la vicenda probabilmente non si risolverà mai fino a fondo, ciononostante essa mostra alcuni fattori importanti concernenti la censura controriformista: l'antagonismo tra l'ambito toscano (i Deputati e il Salviati) e quello veneziano (il Groto), le questioni filologiche (lo scrupoloso lavoro filologico dei Deputati in opposizione alla versione grotiana, la quale tende a trascurare le questioni filologiche) e gli interessi degli editori, che ovviamente vedevano nelle pubblicazioni espurgate di un capolavoro come il Decameron una fonte di guadagno non insignificante.

Un'altra questione pertinente è come mai l'espurgazione di un'opera di tale importanza, dopo la proibizione della rassettatura dei Deputati, fosse affidata ad un letterato poco conosciuto fuori dall'ambito veneziano, che, in opposizione agli espurgatori fiorentini del 1573 , non era competente in filologia. I Deputati, infatti, avevano eseguito sul Decameron un accurato lavoro filologico cercando di ricostruire la lezione originale del Boccaccio e ci riuscirono a tal punto che la loro versione passò per l'edizione più corretta fino a quel momento. ${ }^{3}$ Il Groto, dall'altro lato, non mostrava affatto tali aspirazioni; al proposito è eloquente quanto afferma nella lettera di ringraziamento, rivolta al Padre Paolo della Mirandola, commissario della Santa Inquisizione a Venezia, da cui aveva ricevuto la licenza alla correzione: »vi si richiede più tosto bona volontà, che molta dottrina « (Groto 2007: 265). Da questa affermazione si può già intuire, quale sarebbe stato l'obiettivo principale della sua rassettatura: non certo lo scrupolo filologico. La decisione del Sant'Uffizio di affidare il lavoro di espurgazione al Cieco D'Adria risulta ancora più sorprendente tenendo conto che questi fu per tutta la sua vita interessato alle scienze occulte come l'astrologia, la cabala e la predizione del futuro, la cui pratica, dopo il Concilio di Trento, diventò oggetto di persecuzione. Da giovane (nel 1567, a 26 anni) era stato anche processato per eresia. La sua colpa consisteva nel possesso di alcuni libri proibiti e per questa trasgressione ricevette l'ingiunzione di astenersi per sempre dall'insegnamento, punizione che lo ferì gravemente, visto che l'insegnamento era la sua fonte di guadagno (Mantese 1974: 50). Tra i libri proibiti posseduti dal Cieco spiccavano i Colloquia dell'Erasmo e le opere del Cornelio Agrippa: De vanitate scientiarum, De occulta philosophia e De incertitudine et vanitate scientiarum (Mantese 1974: 44, Rizzi 1987: 39-45). ${ }^{4}$

\footnotetext{
${ }^{2}$ Eloquente, al proposito, la lettera del 10 dicembre 1580, indirizzata a Francesco I dei Medici, in cui dice: "Quanto al Boccaccio pare che ci fossero intrusi altri per intervenirci esso meco, ma resteranno esclusi et andrà innanzi l'ordine di V.A." (P.M. Brown: "I veri promotori della 'rassettatura' del Decameron nel 1582." Giornale storico, CXXXIV, 1975: 314, in: Tschiesche 1987: 243). Il testo non può alludere che all'opera del Groto.

${ }^{3}$ Le cartelle laurenziane conservano l'intera corrispondenza tra i deputati ed il Maestro di Palazzo, padre Maurique. Le lettere mostrano i loro dubbi e indicano i procedimenti e gli obiettivi seguiti, lasciando emergere che il criterio filologico fu d'importanza fondamentale (Cfr. Chiecchi 1984, Sorrentino 1935).

${ }^{4}$ L'Agrippa era stato più volte condannato dalla Chiesa a causa della sua dottrina e delle sue simpatie per il pensiero di Erasmo (Yates 1979: 44-56).
} 
La versione del Groto fu quasi unanimamente riconosciuta come la più bizzarra e la più ardita tra le tre censure in questione; il che si può spiegare con l'ipotesi che per il Groto l'emendazione del Decameron sia stata soprattutto un'occasione per mostrare le sue doti inventive, cercando di riempire narrativamente le lacune prodotte dalla censura (un procedimento dal quale si erano invece astenuti Deputati). Le sue sostituzioni, infatti, non di rado si manifestano nella riscrittura di interi brani e talvolta essi appaiono anche senza riferimento al testo originale (cfr. Chiecchi 1984, Sorrentino 1935). È degno di nota però, dal punto di vista linguistico, la sua aderenza al contesto letterario veneziano, che lo spinge ad inserire nel testo dei brani non motivati dagli obiettivi della censura controriformista, ma piuttosto da personali obiettivi campanilistici. Così, ad esempio, espurga le parti in cui Boccaccio si burla dei veneziani e vi inserisce al loro posto delle valutazioni positive. ${ }^{5}$ Questo antagonismo Firenze-Venezia è però interessante anche su un altro livello, ovvero quale edizione del Decameron prendere come più affidabile per eseguirvi poi gli emendamenti. Se i Deputati usarono come base del loro intervento espurgatorio l'edizione giuntina del 1527, che al momento dell'inizio del loro lavoro rappresentava l'edizione più corretta, il Groto sembra aver utilizzato un'altra edizione, quella a cura di Girolamo Ruscelli, che era uscita nel 1552 a Venezia (Tschiesche 1987: 250). Il Ruscelli, veneziano anche lui, era stato un caro amico del Groto ed è tra l'altro anche l'autore delle note ai margini dell'edizione da lui censurata. Tutti e due facevano parte dell'Accademia dei Pastori Fratteggiani che negli anni '60 si riunivano a Fratta Polesine; fra i partecipanti vi furono anche Lodovico Domenichi, traduttore dell'Agrippa, e Lodovico Dolce, riformatore del Boiardo (cfr. Tschiesche 1987: 83, Rizzi 1987: 39-40, Mantese 1974: 12).

Il Groto, in una lettera del 27 febbraio 1564, addirittura esalta - in uno stile ricco di iperboli - il Ruscelli come »nuovo Prisciano e nuovo Lorenzo Valla della nostra lingua volgare « (Tschiesche 1987: 246), il giudizio non è però troppo motivato: l'edizione a cura del Ruscelli era infatti poco corretta dal punto di vista filologico.

In base a queste informazioni - purtoppo i documenti che spieghino direttamente gli obiettivi del Groto in relazione alla sua espurgazione decameroniana non esistono possiamo supporre che questi abbia letto e di conseguenza trattato il testo boccacciano piuttosto dal punto di vista della »favola« (in termini aristotelici) e non tanto dal punto di vista linguistico.

Malgrado le numerose (e del tutto pertinenti) critiche alla rassettatura grotiana, che la accusano di essere la versione più bizzarra e anche la più mutilata - Sorrentino la chiama addiritura »strazio balordo e ingiustificato dell'arte « e »opera da mattoide « (1935: 203) - l'edizione grotiana godette di un notevole successo ed ebbe più ristampe. E possibile dedurne che la sua opera, seppur priva di intenzioni filologiche, fosse in linea con il gusto del tempo: un gusto tendente all'eccesso e ricco di stravaganze che si afferma in un periodo storico pieno di paradossi. Basterebbe pensare al fatto che l'osservanza dei principi voluti dalla Riforma cattolica coesiste spesso con un vivo interesse per le scienze occulte ed esoteriche.

\footnotetext{
${ }^{5}$ La novella su frate Alberto (IV/2).
} 


\section{RASSETTATURA GROTIANA DEL DECAMERON}

Una domanda che a buon diritto si pone davanti alla rassettatura grotiana è se il lavoro del censore sia stato sorvegliato o no dagli organi dell'Inquisizione. In generale la risposta tende ad essere negativa. Diversamente dal lavoro dei Deputati, che è documentato da una vasta correspondenza con il Maestro del Sacro Palazzo Tommaso Maurique, per gli interventi del Groto non esistono materiali; gli unici documenti pervenutici sono le due lettere di ringraziamento del 18 e 20 gennaio del 1579, una rivolta al Padre Locatelli e l'altra, già menzionata, rivolta al Padre Paolo della Mirandola, in cui il Cieco afferma: »Io prometto d'oprare in guisa, che Iddio ne riceverà Gloria, la chiesa soddisfacimento, il maestro del sacro palazzo diletto, quello autor vita « (Groto 2007: 265). Questa promessa però non dice niente di preciso né sui modi e obiettivi della sua espurgazione, né sulle eventuali richieste del Sant'Uffizio. Sembra dunque che, una volta ricevuto l'incarico, il Groto abbia avuto mani libere di eseguire l'espurgazione a sua discrezione. Analizzando la sua opera d'espurgazione, si può constatare comunque che seguiva in linea massima la prassi più diffusa della censura controriformista, di eliminare rigorosamente qualsiasi riferimento ai rappresentanti della Chiesa cattolica, sostituendoli con i personaggi laici; allo stesso tempo lasciava intatti i brani immorali o lascivi che non toccavano gli ecclesiastici, in opposizione, ad esempio, al Saviati che badava anche all'aspetto morale, inserendo dei cambiamenti che rendevano le storie narrate meno lascive. ${ }^{6}$

La questione della sorveglianza del Groto ci sembra particolarmente importante anche perché questi aveva inserito nella sua versione emendata degli elementi che non solo non hanno niente a che fare con la politica della Controriforma, ma che sono talvolta addirittura in opposizione ad essa: oltre alle già menzionate intenzioni campanilistiche di difesa dell'ambiente veneziano. Ci riferiamo soprattutto agli elementi di astrologia e magia inseriti nei brani espurgati dell'opera boccacciana.

Questi elementi sono osservabili nella sua riscrittura di più novelle, ma qui ci limiteremo ai due casi più eclatanti: la novella di Massetto da Lamporecchio (III/1) e la novella di frate Puccio (III/4). ${ }^{7}$

\subsection{La Novella su Massetto da Lamporecchio}

La famosa novella che narra un caso di immoralità in un monastero femminile, immoralità cui non si sottrae nemmeno la badessa, fu ovviamente censurata in tutte le tre versioni. Nella versione dei Deputati la badessa è trasformata in una vedova che

\footnotetext{
${ }^{6}$ Trasformava ad esempio il ruolo di moglie in ruolo di vedova (Chiecchi 1984: 73).

${ }^{7}$ Groto ha rassettato 46 novelle, 18 delle quali hanno subito solo piccole sostituzioni (per lo più i cambiamenti rigurdano l'indentità dei personaggi) e 14 dei cambiamenti abbastanza estesi, fino al punto che le versioni originarie si rendono talvolta irriconoscibili. (Cfr. Chiecchi 1984: 93). Oltre che nelle novelle sopra menzionate, un richiamo all'astrologia emerge anche nella novella su Alibech (III/10), in cui alla ragazza viene insegnato come "incantare il tempo" da "un giovane per nome Rustico, che per più liberamente poter attendere alla Filosofia, e all'Astrologia, [...] dal consortio humano fuggendo, [...] quivi si riparava.", e nella novella su frate Cipolla (VI/10), in cui il protagonista parla degli alberi del Sole, che "favellano, e rispondono, come noi, e predican l'avenire".
} 
teneva appresso di sé alcune »damigelle povere, et ben nate, et esercitarle in lavori, et ricami, et simiglianti opere femminili« (Chiecchi 1984: 52-53); il Salviati invece traspone la vicenda in Oriente e più precisamente in un serraglio presso Alessandria (Farinelli 2010: 9).

Anche Groto si serve di una trasposizione in un altro ambiente, e cioè in »un altissimo palagio nei boschi«, in cui è chiusa la Prencessa, figlia del Re di Sicilia (in sostituzione della badessa), insieme alle sue compagne (in sostituzione delle monache). Il Groto all'inizio espurga una riflessione boccacciana sulla natura delle monache:

[... che credono troppo bene che] come ad una giovane è sopra il capo posta la benda bianca e indosso messole la nera cocolla, che ella piú non sia femina né più senta de' feminili appetiti se non come se di pietra l'avesse fatta divenire il farla monaca. (Boccaccio 1980: 328) giovani:

Vi inserisce al suo posto una riflessione più generale sulla natura di tutte le donne

[... che credono troppo bene che] una giovane fragile, e delicata, sia di pietra contra gli amorosi appetiti. (Boccaccio 1612: 67)

Siccome il Massetto della versione grotiana non è più »un giovane lavoratore forte e robusto « (329) ma è trasformato in »un de' principali Signori della Toscana, e giovane « (67), si richiede anche l'omissione della riflessione boccacciana sui giovani lavoratori della terra, che nella versione censurata non aveva più fondamento:

E similmente sono ancora di quegli assai che credono troppo bene che la zappa e la vanga e le grosse vivande e i disagi tolgano del tutto a' lavoratori della terra i concupiscibili appetiti e rendan loro d'intelletto e d'avedimento grossissimi. (Boccacio 1980: 329)

Per giustificare perché la figlia del Re dovesse essere chiusa in un luogo talmente solitario, il Groto introduce un'estesa spiegazione:

Dunque belissime Donne, fù già un Re di Sicilia, che una figliuola generò senza più, e veggendola crescere di rare qualità del corpo, e dell'animo, e massimamente d'una singolar bellezza dotata, poi che di dodici anni pervenuta la vide, seco propose di d'intender qual ventura le sovrastasse, e con otto de' suoi principali baroni egli in persona cavalcò verso un Astrologo, che in quei boschi lungi dall'umana conversatione si dimorava, e per le cose da lui ne' tempi adietro predette, s'havea acquistato presso quei popoli grandissima fede. Perciò giunto il Re lo domandò di quel, perche domandare andato vi era, e per conclusione intese, che quantunque i cieli non isforzino, tanto nondimeno inclinano, che se la sua figliuola non fosse stata con diligenza guardata, ella sarebbe suta madre prima, che moglie. Gli otto baroni con Re venuti (ciascun de' quali una figliuola haveva altresi) domandarono e hebbero la stessa risposta, e tornatisi alla Città presero partito di provedervi. Fece dunque il Re in mezo ad alcuni 
solitari, ma dilettevoli boschi un altissimo palagio fondare, d'altissime, e fortissime mura cingendolo, e molto terreno abbracciando, in cui la Prencessa, e le compagne potessero diportarsi, e vietando sotto pena della testa a ciascuno huomo l'entrarvi, fuorche da un castaldo, e ad un giardiniere sì carichi d'anni, che ben sicuramente vi si poteano lasciare [...] (Boccaccio 1612: 67)

In questa sostituzione sono degni di nota, anzitutto, a livello stilistico, l'allontanamento dalla concretezza e dal realismo tipici del Boccaccio. Nella novella in questione, per non compromettere il buon nome del monastero, i dati concreti sono meno presenti che in altre novelle, ma di solito, com'è noto, il Boccaccio ci fornisce non solo il nome preciso dei suoi personaggi, ma anche la provenienza, la professione, lo stato sociale e addirittura il quartiere in cui si vuole vivessero, cercando di localizzare quanto possibile gli avvenimenti in modo da »raccontarli come se questi fossero potuti accadere solamente una volta, in quel dato luogo e con quei dati protagonisti« (Jolles 2003: 104). Nell'inserzione del Groto abbiamo invece dei personaggi molto più indeterminati: "un Re di Sicilia", un Astrologo" "un de' principali Signori della Toscana", aspetto che trova corrispondenza con lo stile tipico delle fiabe, mirante a una decontestualizzazione dei fatti narrati (cfr. Jolles 2003: 126). Nella sua versione già l'inizio della novella - "fu già un Re di Sicilia" - presenta una formula tipicamente fiabesca e fiabesca è anche la trama, che prevede la visita dall'Astrologo, la sua predizione, lo sforzo del padre per evitare il compiersi del destino, il fallimento ineluttabile dell'impresa, visto che la predizione si avvera.

In realtà nell'Italia del Quattrocento l'evoluzione del gusto letterario aveva comportato negli scrittori una minore frequentazione della novella, un genere tendenzialmente basato sulla rappresentazione realistica dei fatti. Auerbach (1984: 36) afferma che si preferiva »non cercare più l'unione delle cose terrene nella prosa realistica, ma piuttosto confonderle fiabescamente nella poesia«; cominciavano dunque a fiorire i poemi cavallereschi di Pulci, Boiardo e infine Ariosto. La ripresa di un interesse per la novella avviene solo nel Cinquecento con i novellieri più noti, Matteo Bandello e Giraldi Cinzio. Anche quest'ultimo, nel suo Discorso attorno al comporre dei romanzi (1554), esalta il genere del poema cavalleresco e loda lo stile del Boiardo e dell'Ariosto. In Italia la prima raccolta di fiabe appare nel 1550, quando Giovanni Francesco Straparola pubblica le sue Piacevoli notti. Perciò possiamo ipotizzare che sia stato anche il gusto letterario dell'epoca a indurre Groto a inserire elementi fiabeschi dentro l'opera boccacciana, operazione comportante un effetto opposto a quello voluto dal Boccaccio, il quale tendeva invece, come noto, a una rappresentazione realistica delle vicende.

Non è da trascurare nemmeno la considerevole somiglianza di questa trama con quella di un'altra opera grotiana, la tragedia Dalida, pubblicata nel 1572 (Tschiesche 1984: 251). Essa narra una storia simile, e precisamente quella della una figlia di un Re che il padre rinchiude in un palazzo in mezzo ai boschi per »conservarle e la vita, e l'onore «. Anche in tal caso le precauzioni del padre non possono prevenire che il giovane (nipote e futuro assassino del padre della giovane) entri nel palazzo e di seguito nel cuore della figlia del Re: 
$[\ldots] \mathrm{E}$ tra le selve in un palagio

La chiusi in compagnia d'altre Donzelle,

A cui fuor, che l'uscir non mancasse altro.

Ma si inganna quel padre, il quale stima

L'onestà della figlia intatta, e salva,

Per haverla rinchiusa in grembo à i marmi,

E di ferro, e d'acciar cinta d'intorno,

Quando ella in caste voglie il cuor non chiude. (Groto 1572: 10).

Analoghi sono dunque i personaggi (il Re e sua figlia, del tutto assenti nell'originale boccacciano) come pure l'elemento della preoccupazione paterna per la castità della figlia, proccupazione smisurata fino al punto di fargli prendere provvedimenti oltre modo severi, come quello di isolare la figlia da possibili contatti con l'esterno. La trama del Groto fu commentata anche dal Ruscelli, autore delle glosse in margine della versione grotiana, che però trovò problematico solo il fatto che »il Re consignasse per stanza alla figliuola un luogo così solitario senza custodia di persona, che di lei cura havesse « (Boccaccio 1612: 67).

$\mathrm{Nel}$ seguito il Groto tende a seguire il testo originale ed interviene soltanto per effettuare le sostituzioni dei personaggi già menzionati (monache - donzelle, Massetto - il giovane) e operare alcune omissioni che sono la conseguenza di tali sostituzioni (ad es. il passo in cui le monache discutono di aver promesso la verginità a Dio). Aggiunge però un elemento nuovo in relazione al giovane protagonista: se nella versione originale Massetto si fa muto per poter entrare nel monastero, a Groto ciò non basta - il suo protagonista non si fa solo muto, ma si tinge anche la barba e i capelli di bianco, ovviamente per sembrare più vecchio e di conseguenza meno attraente alle giovani donzelle (dato che, nella versione grotiana, non è più un semplice lavoratore ma un gran signore e di conseguenza più capace di suscitare fascino nelle giovani). Questa aggiunta, che dal punto di vista degli obiettivi della censura controriformista non era per niente necessaria, mostra lo sforzo del Groto di emendare il testo in modo tale che potesse risultare il più convincente possibile la logica dei fatti; voleva dunque, un protagonista e una trama convincenti, obiettivo che gli è in generale tristemente fallito, ma che nondimeno dimostra il suo approccio verso la letteratura e, in senso più stretto, verso i procedimenti della censura che, secondo lui, non avrebbe dovuto limitarsi all'eliminazione di passi incriminati, ma riempire narrativamente le lacune.

Un cambiamento rilevante avviene di nuovo verso la fine della novella, dove viene eliminato il colloquio tra Massetto e la badessa, oltre che nella conclusione che Groto rende pì corta e sintetica:

[... rotto lo sciliguagnolo], con gran maraviglia di lei la fece accorta di ciò era infin all'hora avvenuto. In questo mezo il Re, e gli altri Baroni vollero saper dall'Astrologo se 'l reo influsso ancora passato fosse, e egli rispose, che era passato, ma che tanto ne era avvenuto, quanto ne havea predetto. Questo inteso, andarono tutti insieme al palagio, e trovate le figlie gravide, e udito di chi, lo fecero prendere, minacciandogli una acerbissima morte. Ma il giovane Signore veggendosi in tanto pericolo, e havendo compassion di se stesso, promise di sposar la Prencessa, e di far che altre ancora 
sarebbono da suoi baroni sposate, alche s'accordarono i padri, e così si fece, celebrandosi le nozze solenni, alle quali fu chiamato lo Astrologo, e egli vi venne motteggiando le giovani dolcemente, le quali hebbero più ventura, che senno. (Boccaccio 1612: 68-69)

Come succede anche in altri casi, ${ }^{8}$ Groto sostituisce qui l'originale dialogo tra i protagonisti, che è un modo narrativo tipico per il Boccaccio in quanto permette ai personaggi di fare sfoggio di retorica e raggiungere così i loro obiettivi, con un passo di sole azioni. La conclusione della novella (in particolare le nozze del protagonista e dei suoi otto baroni), funzionerebbe molto meglio se facesse parte di una fiaba, mentre dentro a un testo boccacciano, che mira alla rappresentazione realistica dei fatti, risulta strana, inverosimile e poco convincente.

\subsection{La novella su don Felice e frate Puccio}

Un'altra novella in cui appare l'astrologia e l'arte di predire l'avvenire è la quarta novella della terza giornata; per fare una breve sintesi della versione censurata basti riferire il titolo emendato: »Felice insegna a Puccio come egli diverrà Astrologo, e indovino, facendo una sua arte; la quale Puccio facendo, Felice in questo mezzo con la moglie di lui si dà buon tempo« (Boccaccio 1612: 73).

Puccio dunque viene trasformato da un bizzocco che »usava molto la chiesa, [...] diceva suoi paternostri, andava alle prediche, stava alle messe« (1980: 361) a un sempliciotto che »era vago di udire, e di raccontar le nove del mondo, $[\ldots]$ ma sopra tutto dilettavasi di indovinare i successi avvenire, $[. .$.$] e perciò si vedea sempre con Lunari,$ con Pronostichi, con Tavole, e con Effemeridi in mano«; Felice, invece, viene trasformato da monaco a scolare che si finge »grandissimo conoscitor delle stelle« (1612: 73).

In questa novella le scienze occulte diventano una sostituzione per la penitenza che Puccio effettua per diventare beato; del resto anche il Salviati si era servito di una soluzione simile: nella sua versione Felice si finge alchimista e studioso di testi esoterici (Chiecchi 1984: 81). C'è una differenza fondamentale che distingue questa novella da quella precedentemente considerata. Nella novella su Massetto il ruolo dell'Astrologo viene preso sul serio, le sue predizioni (malgrado lo sforzo del padre di impedirlo) si realizzano, il che dà alla novella un carattere fiabesco, visto che tali avvenimenti di solito vengono narrati nelle fiabe nelle quali l'inverosimiglianza è attesa. Nella novella di Puccio l'astrologia ha invece una funzione completamente diversa: è usata sostanzialmente come mezzo per beffare il protagonista e non viene presentata come una scienza seria, veramente capace di predire l'avvenire. Nella versione originale la vicenda di Puccio viene commentata dal narratore con un'ironia eloquente: »assai persone sono che, mentre essi si sforzano d'andarne in Paradiso, senza avvedersene vi mandano altrui« (1980: 360) e Groto trasforma la frase con un'ironia per niente inferiore: »assai persone sono che, mentre essi si sforzano di veder le cose avvenire, le presenti non veggiono« (1612: 73).

\footnotetext{
${ }^{8}$ Il caso più eclatante è la novella su Ser Ciappelletto, che viene privata della confessione e la lacuna viene riempita esclusivamente con gli elementi d'azione.
} 
Questo passo è interessante perché mostra una posizione di un distacco ironico verso l'astrologia, una scienza che il Groto nella vita privata invece prendeva molto sul serio; tra l'altro scrisse una Chiromantia (inedita) e concepì anche una Effemeride con le previsioni dal 1583 al 1600 (Rizzi 1987: 47). Del resto anche le Accademie di cui faceva parte mostravano un vivo interesse per l'occulto, per le scienze ermetiche e cabalistiche, secondo una tendenza diffusa in tutto il Cinquecento (cfr. Yates 1979, Thorndike 1941). Sembra dunque che l'ironia sull'arte della predizione, nella novella censurata di Puccio, non presenti l'opinione personale del Groto, bensì nasca dalla trama stessa e ricalchi la battuta che appariva nell'originale. Non sfugge tuttavia che, dove il Groto sostituisce il passo con le istruzioni sul come fare penitenza con uno in cui dà istruzioni concrete su come imparare a predire l'avvenire, introduce anche alcune informazioni generali sull'astrologia, quasi per sottolienare la sua competenza in materia:

[...] si come di giorni, e di mesi è composto l'anno commune, così di anni communi è composto l'anno grande, ilquale a punto per tua grandissima ventura comincia questa medesima sera. Quest'anno grande non è già di tanti anni, quanti fin qui si dissero. Ma si è per prova trovato, esser solo di anni quaranta de nostri. Et si come anticamente (perchè hoggidì per la varietà de' tempi, questa regola è rimasa fallita) da' primi giorni di Gennaio si conoscevano, e pronosticavano gli effetti di tutti i Mesi di quell'anno, servendo un giorno ad un mese, così dalle prime quaranta notti di questo anno grande, che questa sera havrà il suo principio, si raccolgono tutti e publichi, e particolari effetti de gli anni quaranta a venire, i quali aviso, che ti sien per bastar tutto il rimanente della tua vita. Percioche le stelle minutamente osservate di notte in notte, vengono mostrando gli influssi di anno in anno [...] (Boccaccio 1612: 74)

Girolamo Ruscelli nelle sue note al margine spiega il significato dell'espressione »anno grande « e scrive: »il nono Cielo co'l suo proprio moto si rivolge dall'Occidente in Oriente nello spazio d'anni trentasei mille secondo Tolomeo; ilqual corso di tempo è detto anno Platonico, ovvero anno grande.« (Boccaccio 1612: 74). Il passo grotiano accenna dunque al sistema solare geocentrico tolemaico. Si osservi en passant che un interesse particolare per gli studi astronomici di impostazione tolemaica lo coltivava anche Giovanni Maria Bonardo, amico di Groto e membro dell'Accademia dei Pastori Fratteggiani; questi era autore dell'opera La grandezza, larghezza e distanza di tutte le sfere, pubblicata a Venezia nel 1584, opera che alcuni studiosi credono fosse in realtà stata scritta prevalentemente dal Groto (Mazzetti 1987: 82, 92).

Tenendo conto, da una parte, dell'effettivo interesse del Groto per l'astrologia e l'arte di predire il futuro e, dall'altra, del fatto che questo brano è inserito in un testo che presenta una beffa, dobbiamo chiederci se l'effetto ironico non vada un poco contro le convinzioni del Groto e trovi un suo senso soprattutto nella sua ricerca di rispettare (almeno in questo caso) la logica narrativa boccacciana. La novella censurata viene dunque da lui conclusa con una battuta di ironia arguta su chi crede in questa scienza. Ed è un'ironia pari a quella boccacciana, fenomeno altrimenti raro nella sua rassettatura del Decameron: 
[...] avvenne che dove frate Puccio faccendo penitenza si credette mettere in Paradiso, egli vi mise il monaco. (Boccaccio 1980: 367)

[...] avvenne che dove frate Puccio pensò di contemplar le corna della nova Luna nel Cielo, altri gliele pose sul capo. (Boccaccio 1612: 75)

E ancora nel passo conclusivo:

E quando portò a Felice le cose ch'egli havea scritto, lo Scolare si dolse, affermandoli che non havea scritto bene, e che esso havea fatto meglio assai quelle notti giacendo in letto. (Boccaccio 1612: 75)

Da quest'ultima frase risulta in modo ancora più palese la portata della beffa giocata al povero Puccio: non solo lo scolaro »gli pose le corna sul capo«, ma anche tutta la sua fatica delle ultime quaranta notti gli risultò vana, visto che dai suoi appunti non avrebbe potuto predire l'avvenire. Qui il Groto, per quanto riguarda la portata della beffa, fa un passo ancora più avanti di Boccaccio: se il Puccio boccacciano non viene a sapere se la sua penitenza lo avrebbe portato in Paradiso e così alla fine della novella tutti i protagonisti sono contenti perché ognuno ha quello che voleva, il Puccio di Groto rimane profondamente deluso.

\section{CONCLUSIONE}

Cercando di inquadrare gli interventi relativi alla novella su frate Puccio nel contesto più generale delle operazioni effettuate dal Groto sul capolavoro di Boccaccio, occorre rilevare che questo rifacimento produce, malgrado le trasformazioni e sostituzioni subite, un effetto più o meno paragonabile a quello del testo originale, che è un fenomeno riscontrabile in ben poche novelle censurate dal Groto. Non di rado, infatti, questi interviene in maniera così radicale sul testo, che la trama originale viene completamente stravolta; in altri casi invece, pur cercando di seguire la trama originale, deforma e appiattisce gravemente, con le sue trasformazioni, il significato della novella, oltre a creare enormi dissonanze stilistiche poiché inserisce brani di gusto manierista che si scontrano con lo stile di Boccaccio. Come abbiamo visto nel caso della novella su Massetto da Lamporecchio, tali inserzioni provocano un effetto bizzarro a causa dell'incompatibilità tra due modi di narrare: l'uno verosimile e ironico e l'altro improntato al meraviglioso e al fiabesco e teso al moralismo (visto che tutte le donzelle, inclusa la Prencessa, alla fine vengono maritate per non vivere più nel peccato). Si tratta di una conclusione lontanissima da quella dell'originale, in cui il protagonista, con l'aiuto del proprio ingegno, riesce non solo a »adoperare bene la sua giovinezza«, ma anche ritornare a casa ricco e contento.

La novella del Puccio, invece, anche con le sostituzioni che presenta, è profondamente diversa: l'ironia boccacciana non viene diminuita, bensì trasformata in modo da ottenere un effetto paragonabile. Assai meno riuscite, ad esempio, risultano le sostituzioni nella novella di Alibec e Rustico (III/10), in cui alla ragazza viene insegnato come calmare la tempesta, e quelle nella novella di Martellino (II/1), in cui il protagonista finge di essere femmina - soluzioni che risultano molto bislacche e dove vanno perse 
quasi completamente l'argutezza e l'ironia dell'originale. Forse è più che altro una felice coincidenza che l'astrologia risulti, nella novella su Puccio, come una felice alternativa alla tematica religiosa della versione originale (la previsione di premi e penitenze dopo la morte); tanto la dura penitenza che avrebbe dovuto portare il protagonista in Paradiso quanto la veglia fatta con l'intenzione di predire l'avvenire e ottenere pregi e fama funzionano infatti bene come mezzi attraverso cui burlarsi dell'ingenuità della gente semplice che crede di poter raggiungere in quaranta notti dei risultati che cambino la loro vita, terrena o oltremondana.

Difficile credere che il burlarsi dell'arte di predire il futuro fosse anche attitudine personale del censore, al quale invece questa disciplina stava molto al cuore. La ragione per cui aveva comunque deciso di usarlo come elemento narrativo, seguendo per una volta la trama del Boccaccio, sta forse altrove. La digressione in cui inseriva nozioni sull'anno grande platonico e sulle stelle dalle quali si sarebbe potuto, osservandole minutamente, predire gli eventi futuri, gli permetteva di mostrare i suoi interessi personali e di condividere il suo sapere con gli altri. Se si rimuove il contesto boccacciano in cui il richiamo alle previsioni astronomiche si riveste di beffa, quello che rimane è proprio l'intenzione, non ironica, di diffondere il suo sapere in materia.

\section{Ljubljana, Slovenia}

\section{BIBLIOGRAFIA}

Auerbach, Erich. Zur Technik der Frühreneissancenovelle in Italien und Frankreich. Heidelberg: Carl Winter Universitätsverlag, 1971 (trad.it.: La tecnica di composizione della novella. Roma: Theoria, 1984).

Boccaccio, Giovanni. Decameron. A cura di Vittorio Branca. Torino: Einaudi, 1980. . Il Decamerone di messer Giovanni Boccaccio cittadin fiorentino. Di nuovo riformato da M. Luigi Groto Cieco d'Adria con permissione de'Superiori. Et con le dichiarationi, avertimenti, \& un vocabolario fatto da M. Girolamo Ruscelli. Con privilegi. Venezia: Farri, 1612.

Chiecchi, Giuseppe e Luciano Troisio. Il Decameron sequestrato. Le tre edizioni censurate nel Cinquecento. Milano: UNICOPLI, 1984.

Groto, Luigi. Le Famigliari del Cieco d'Adria. Ed. Marco de Poli, Luisa Servadei e Antonella Turri. Treviso: Antilia, 2007.

La Dalida tragedia nova di Luigi Groto Cieco di Hadria. In Venetia, 1572.

Farinelli, Patrizia. "Cose turche nella censura del Decameron a cura di Salviati." Italien und das Osmanische Reich. Herne: G. Schäfer, 2010: 241-267.

Jolles, André. I travestimenti della letteratura: saggi critici e teorici (1897-1932). Ed. Silvia Contarini, premessa di Ezio Raimondi. Milano: Bruno Mondadori, 2003.

Mantese, Giovanni. Due processi per eresia: la vicenda religiosa di Luigi Groto, il Cieco di Adria e della nobile vicentina Angelica. Vicenza, Officine grafiche STA, 1974.

Mazzetti, Adriano. "L'ambiente culturale rodigino tra Cinque e Seicento. Le accademie." Luigi Groto e il suo tempo: atti del Convegno di studi, Adria, 27-29 aprile 1984. A cura di Giorgio Brunello e Antonio Lodo. Rovigo: Minelliana. 1987: 79-118.

Rizzi, Franco. »Le socialità profonde: la famiglia di Luigi Groto il Cieco d'Adria. « Luigi Groto e il suo tempo: atti del Convegno di studi, Adria, 27-29 aprile 1984. Ed. Giorgio Brunello e Antonio Lodo. Rovigo: Minelliana. 1987. 23-60. 
Rozzo, Ugo. La censura libraria nell'Europa del secolo XVI. Convegno internazionale di Studi, Cividale del Friuli 9/10 Novembre 1995. Udine: Forum, 1997.

. La letteratura italiana negli Indici del Cinquecento. Udine: Forum, 2005.

Yates, Frances. The Occult Philosophy in the Elisabethan Age. Londra e New York: Routledge, 1979.

Sorrentino, Andrea. La letteratura italiana e il Sant'Uffizio. Napoli: Perella, 1935.

Thorndike, Lynn. A history of magic and experimental science. Volumes $V$ and V, The sixteenth century. New York: Columbia University Press, 1941.

Tschiesche, Jacqueline. "Il rifacimento del Decamerone di Luigi Groto." Luigi Groto e il suo tempo: atti del Convegno di studi, Adria, 27-29 aprile 1984. Ed. Giorgio Brunello e Antonio Lodo. Rovigo: Minelliana, 1987: 237-269. 